\title{
Traitement androgénique substitutif et cancer prostatique
}

\author{
Thierry FLAM \\ Clinique Urologique, Hôpital Cochin, Paris
}

\section{RÉSUMÉ}

Les études épidémiologiques ont montré que le risque de cancer de la prostate n'est pas corrélé au taux sérique de testostérone, les androgènes ne semblent pas pouvoir induire un cancer de la prostate chez l'homme. Par contre, l'apport exogène de testostérone peut démasquer un cancer prostatique jusque là freiné par un hypogonadisme. La détection d'un cancer latent avant le début du traitement doit suivre les règles habituelles fondées sur le toucher rectal et le taux sérique du PSA. Une surveillance semestrielle est justifiée en début de traitement, ensuite, la surveillance doit être annuelle.

Mots clés : cancer, prostate, dépistage, testostérone, PSA

\section{DONNÉES ÉPIDÉMIOLOGIQUES}

Les données épidémiologiques apportent des informations sur les rapports éventuels entre l'imprégnation androgénique et les pathologies prostatiques. On observe avec l'âge une diminution de la testostéronémie. Dans le même temps, le volume prostatique augmente (hyperplasie bénigne de la prostate), les troubles mictionnels augmentent, le taux sérique de PSA augmente et l'incidence du cancer de la prostate augmente. Ces données, paradoxalement, vont plutôt contre l'idée d'un effet général délétère des androgènes sur la prostate.

\section{ANDROGÈNES ET PROSTATE}

Les androgènes sont nécessaires au développement initial de la prostate, et le déficit en dihydrotestostérone entraîne l'absence de développement prostatique. Cependant, les androgènes semblent ensuite avoir un rôle tout à fait passif vis-à-vis de la prostate.

Les anti-androgènes, utilisés couramment en pratique clinique, entraînent une diminution du volume prostatique (environ 30\%), une diminution du taux sérique du PSA, et une amélioration des symptômes mictionnels. La suppression androgénique est utilisée dans le traitement palliatif du cancer de la prostate.

Le traitement androgénique substitutif ramène le taux sérique de testostérone et le taux de PSA à la normale. En cas de déficit congénital, le volume prostatique double en six mois. En cas d'hypogonadisme acquis, le traitement ramène le volume prostatique à la normale $[1,8]$.

\section{ANDROGÈNES ET CANCER DE LA PROSTATE}

Les études épidémiologiques ont montré que le risque de cancer n'est pas corrélé au taux sérique de testostérone [3, 5].

Chez l'animal, le cancer prostatique peut être induit par les androgènes [10]. Par contre, les androgènes ne semblent pas pouvoir induire un cancer de la prostate chez l'homme $[9,6]$.

Un cancer existant est stimulé par les androgènes [7], et surtout l'apport exogène de testostérone peut démasquer un cancer prostatique jusque là freiné par un hypogonadisme [4]. Le niveau de confiance des études épidémiologiques publiées doit cependant être tempéré par le fait que

Correspondance : Professeur Thierry FLAM, Clinique Urologique, Hopital Cochin, 27 rue du Fbg Saint Jacques, 75014 Paris. 
le cancer prostatique est très fréquent dans la population cible du traitement substitutif, et que l'incidence du cancer « latent » est d'environ $30 \%$ chez les sujets au-dessus de 60 ans. Des études incluant des effectifs considérables et un suivi prolongé seront donc indispensables.

Jusque là, les publications concernant les effets du traitement substitutif sur le cancer de la prostate ont concerné des cas anecdotiques ou des séries limitées chez des patients jeunes. Le traitement substitutif prolongé, évalué sur une population de 11 patients suivis au maximum 10 ans, ne semble pas entraîner de modification du PSA, d'augmentation du PSA au-dessus de la normale, de troubles mictionnels, et n'augmente pas les indications des biopsies prostatiques [2].

\section{RECOMMANDATIONS}

- Les patients ayant un traitement substitutif sont des patients dont le risque « prostatique » est celui de la population normale du même âge ;

- La détection d'un cancer latent avant le début du traitement doit suivre les règles habituelles fondées sur le toucher rectal et le taux sérique du PSA. Il n'y a pas lieu de faire des biopsies prostatiques systématiques ;

- Une surveillance particulière, semestrielle, est justifiée en début de traitement (en raison du risque de cancer « masqué » éventuel) ;

- Ensuite, la surveillance (toucher rectal, dosage sérique du PSA) doit être annuelle, et d'éventuelle biopsies prostatiques décidées selon les règles habituelles ;

- Un cancer avéré est une contre-indication formelle à l'initiation et/ou à la poursuite du traitement.

\section{RÉFÉRENCES}

1. BEHRE H.M., BOHMEYER J., NIESCHLAG E. ; Prostate volume in treated and untreated hypogonadal men in comparison to age-matched controls. Clin. Endocrinol., 1994, 40 : 341-349.

2. BEHRE H.M., VON ECKARDSTEIN S., KLIESCH S. et al. : Long-term substitution therapy of hypogonadal men with transscrotal testosterone over 7-10 years. Clin. Endocrin., 1999, 50 : $629-635$.

3. CARTER H.B., PEARSON J.D., METTER E.J. et al. : Longitudinal evaluation of serum androgen levels in men with or without prostate cancer. Prostate, 1995, 27 : 25-31.

4. CURRAN M.J., BIHRLE W. : Dramatic rise in prostate specific antigen after androgen replacement in a hypogonadal man with occult adenocarcinoma of the prostate. Urology, 1999, $53: 423$ 424.

5. HULKA B.S., HAMMOND J.F., DIFERNANDO G. et al. : Serum hormone levels among patients with prostatic carcinoma or benign prostatic hyperplasia and clinic controls. Prostate, 1987, $11: 171-182$.

6. ISAACS J.T. : Role of androgens in normal and malignant growth of the prostate. In : Bhasin S. ed. Pharmacology, Biology, and Clinical applications of Androgens. New York, Wiley- Liss, 1995: 95-100.

7. J ACKSON J.A., WAXMAN J., SPIEKERMAN A.M. : Prostatic complications of testosterone replacement therapy. Arch. Intern. Med., 1989, 149 : 2365-2366.

8. MEIKLE A.W., ARVER S. DOBS A.S. et al. : Prostate size in hypogonadal men treated with a nonscrotal permeation-enhanced testosterone transdermal system. Urology, 1997, 49 : 191-196.

9. MONTIE J.E., PIENTA K.J. : Review of the role of androgenic hormones in the epidemiology of benign prostatic hyperplasia and prostate cancer. Urology, 1994, $43: 892-899$

10. NOBLE R.L. : Development of prostatic adenocarcinoma in $\mathrm{Nb}$ rats following prolonged sex hormone administration. Cancer Res., 1977, $37:$ 1929-1933.

ABSTRACT

\author{
Androgen replacement therapy and \\ prostatic cancer
}

Thierry FLAM

Epidemiological studies show that the risk of prostate cancer was not related to testosterone blood levels. Androgens do not seem to be able to cause prostatic cancer in men. However, administration of exogenous androgens can unmask a prostatic cancer that was, until then, hidden by hypogonadism. Screening of a latent prostatic cancer before the induction of an androgenic treatment must follow the usual recommendations based on digital rectal examination and PSA level determination. A special attention is to be paid when beginning an androgen replacement therapy with a control at six months; thereafter a follow up including digital rectal examination and PSA must be performed every year.

Key Words : cancer, prostate, screening, testosterone, PSA 